\title{
Extremes and Moderates: A characterization and an application to lobbying
}

\author{
Murali Agastya \\ Economics Discipline, SEPS \\ University of Sydney \\ Sydney NSW 2006 \\ AUSTRALIA \\ m.agastya@econ.usyd.edu.au
}

\author{
Kunal Sengupta \\ Economics Discipline, SEPS \\ University of Sydney \\ Sydney NSW 2006 \\ AUSTRALIA \\ k. sengupta@econ.usyd.edu.au
}

8 December, 2003

\begin{abstract}
In a society where individuals differ in their evaluation of different social policies, when might one consider a given individual as having preferences that are "extreme" relative to the others'? And how important are such preferences in determining eventual policy?

In this paper, we describe an individual as being "extreme" if her views differ from the mainstream to the extent that the rest of the society is able to unanimously agree on a compromise policy that they strictly prefer to what might have been the outcome if such an individual has her own way.

Relying on the intermediate property of preferences due to Grandmont 1978 we provide a simple geometric characterization of "extreme preferences". Furthermore, we also present an illustrative positive model of lobbying activity where we apply our characterization result to show that every equilibrium social policy is determined only by the activities of those holding "extreme preferences" even when they are a minority.
\end{abstract}

Keywords: extremes, moderates, intermediate preferences, collective decisions and lobbying.

\section{INTRODUCTION}

Consider a society of individuals who differ in their ranking of various social policies. Which of these individuals exhibit preferences that are extreme relative to others? And how important are such preferences in determining eventual policy?

Referring to someone as having "extreme views" is of course common in everyday rhetoric. An increasing number of formal works in economics and political science also use the labels "extremes" or "moderates" in their work, particularly in relating the pattern of collective decisions with individual characteristics. Banerjee and Somanathan 2001, Esteban and Ray 2001, Osborne et al. 2000, Esteban and Ray 1999 and Lohmann 1993 are a few representative examples of this section of the literature. However, we are yet to come across any formal treatment or discussion of what it means for an individual to have "extreme" preferences or views. 
This paper studies environments involving collective decisions. We propose a simple and intuitive definition of when an individual can be regraded as having "extreme preferences" and characterize them in simple geometric terms. We also present an illustrative positive model of collective decision making where any equilibrium social policy is determined only by such individuals even if they constitute a minority.

In common parlance, one sense in which the word "extreme" is used is to express the sentiment that the person(s) in question "holds views that differ drastically and fundamentally from those in the rest of the society". It is this sense of the word that our formal definition seeks to capture. The basic presumption here is that if someone were to so "differ" from the mainstream, the rest of the society should then be able to agree on a compromise policy that they unanimously (and strictly) prefer to what might have been the outcome if an individual with extreme views has her way.

To describe this formally, we view a society as consisting of a set of feasible social policies $X$ and a family of utility functions $\{u(\cdot, t)\}_{t \in T}$ indexed by elements of a set $T$ where each member of the family ranks different policies in $X$. Assume that for each type $t$ in $T$, there is a unique policy $\phi(t)$ in $X$ which $t$ ranks the highest among all other feasible policies. We call $\phi(t)$ the favorite policy of $t$.

Definition (Extreme Types). Given a society $\left(X,\{u(\cdot, t)\}_{t \in T}\right)$ the preferences corresponding to a type $t$ in $T$ are said to be extreme (or that $t$ is an extreme type) if there exists a compromise policy $x$ that is feasible and all other types in $T$ strictly prefer $x$ to $\phi(t)$. When a type is not extreme, we say that it is moderate.

According to our definition, existence of a single compromise policy against a given type is adequate for her to be called extreme even though it is possible that the preferences of the different types are the same except for their ideal policies. One may then argue that the deviation from the views of others is not drastic. This however is not so. It will be evident from our results that innocuous assumptions (such as the policy space be sufficiently rich, i.e. have a non-empty interior and continuity of preferences) will ensure that when a type is extreme, there is an open set of policies each of which can be chosen as a compromise against it. In this sense an extreme type according to our definition is also fundamentally different from the rest of the society.

A further salient feature of our definition is worth flagging at the outset. Note that whether a type is extreme or not depends only on the set of types $T$ that are present in the society and not on its distribution. It is thus entirely possible for a type to be extreme even when the individuals of this type constitute a majority in the population. We do not consider this to be a problem. Since our primary interest here is to specify an intrinsic property of the different "views" in terms of their relationship to each other - the information on who and how many of them subscribe to this particular preference ordering seem to us of secondary importance.

This approach of describing extremes independently of their distribution is also attractive in so far as it allows one to talk about extremes and moderates independently of the collective decision making process that is being used by the society under consideration. The characterization results of extreme types that we obtain can in fact be used to discuss which collective decision schemes favor manipulation by extremes. The lobbying models presented in Section 3 are a case in point. 
A preliminary observation that deserves emphasis is that our intuitive definition of 'extreme preferences' certainly agrees with the informal use of the terms extremes and moderates in the literature cited earlier. For, in these and several other spatial models of voting, the preferences in the society are a finite family of single peaked preferences ordered on a line, such as those considered by Black [1958. In these environments, it is easy to see that the types furthest to the left and to the right are the only extreme types according to our definition 1 . Our results, Theorem 1 and Theorem 2 generalize the above feature that an extreme type cannot be expressed as a convex combination of the other types to higher dimensions.

We consider societies where $X$ and $T$ are subsets of some (possibly different) Euclidean space and the preferences satisfy the intermediate property of Grandmont [1978, a natural extension of the linearly ordered single peaked preferences to higher dimensions. In Theorem 1 . we show that when there are finitely many types a type $t \in T$ is extreme if and only if the following hold: a). $t$ is not a convex combination of the remaining types, $b) . \phi(t)$ is not the favorite policy of any type who is an average of the remaining types. This result of course is consistent with the observation that the end points are the extreme types if types are unidimensional as in the standard literature.

When the society consists of an infinite family of preferences, then extreme types may not exist. In Section 2.1 we consider a natural weakening of the definition of extreme types. Theorem 2, an analogue of Theorem 1 then obtains.

The usefulness of our characterization result is explored in Section 3. There we study a variant of a lobbying model that has been extensively studied in the literature on rent seeking at least since Tullock 1980. (See also the references listed in Esteban and Ray 1999]). We show (Theorem 3) that economies with a large (but finite) number of individuals, in every equilibrium, the individuals who actively lobby are necessarily of extreme type. Since we do not make any assumptions on the distribution of types, the result also highlights the importance of those with extreme views.

Also of independent interest is a corollary to Theorem 3 which shows that with unidimensional type space, the only types that lobby are those at the two end points. In Esteban and Ray [1999, the total amount of resources spent on lobbying activities is taken as a measure of conflict in the society. One of their comparative statics results is that this societal conflict is maximized when there are two groups whose members have extreme preferences. This "twin peaks" equilibrium which maximizes conflict and polarization appear endogenously in our model. This difference can be attributed to the fact that in our model, it is the individual agent (and not the group) which chooses its level of lobbying activities.

The rest of the paper is organized as follows. Section 2 develops the background and contains the result for a finite family of preferences, namely Theorem 1. Generalization of this result to infinite family of preferences is done in Section 2.1. Finally, Section 3 contains results on the lobbying model.

\section{The Intermediate Property and Extreme Preferences}

In the sequel (with the exception of Example 1), the set of policies $X$ is a compact and convex subset of $\mathbb{R}^{k}$, the set of types $T$ is a compact subset of $\mathbb{R}^{\ell}$ and $u: \mathbb{R}^{k} \times \mathbb{R}^{\ell} \longrightarrow \mathbb{R}$ describes

\footnotetext{
${ }^{1}$ We discuss this case in detail in the next section
} 
the preferences. That is $u(x, t)$ is the utility of policy $x$ for a type $t$. Throughout, we assume that for each $t \in T, u(\cdot, t)$ is continuous and strictly quasi-concave. This in particular implies that $\phi(t)$ is well defined. Let $(X, T, u)$ denote a typical society.

In many models of conflict and spatial models of voting behavior, it is common to restrict attention to societies where $T, X \subseteq \mathbb{R}^{\ell}$ and $u(x, t)=f(\|x-t\|)$ for a strictly decreasing concave real valued function $f(\cdot)$. Then $\phi(t)$ is the element of $X$ that is closest to $t$. As the utility for any policy depends only on the Euclidean distance, we shall refer to such a society as an Euclidean society of dimension $\ell$.

A somewhat different environment that fits our framework is the one studied by Banerjee and Somanathan 2001 in their "model of voice". In that model, the policy space is $X=[0,1]$ and there are two states 1 and 2. If a policy $x$ is chosen, the vNM utility of each individual is $f(x)$ if state 1 occurs and $f(1-x)$ if state 2 occurs. Individuals however differ in their assessments of the likelihood, say $t$, of state 1. Each individual provides advice to a leader who then makes a collective choice by choosing an element of $X$. The utility function of a type $t$ individual when policy $x$ is chosen is then given by $u(x, t)=t f(x)+(1-t) f(1-x)$ where $t \in T=[0,1]$. When $f(\cdot)$ is concave, it is possible obtain $\phi(t)$ for every $t$. One can then use our definition to determine which types $t$ have 'extreme' beliefs. It is however important to point out that much of our intuition for the characterization of extreme types is derived from considering Euclidean societies.

For an arbitrary society with at most two types, each of them is trivially extreme. When there are more than two types however, matters are more complicated. In fact, it is possible to have societiesq where no type is extreme. As a simple example, consider the case where $X=\left\{x_{1}, x_{2}, x_{3}, x_{4}\right\}$ and $T=\left\{t_{1}, t_{2}, t_{3}, t_{4}\right\}$. The four different strict rankings of these policies corresponding to the different types are shown Table 1.

\begin{tabular}{cccc}
$t_{1}$ & $t_{2}$ & $t_{3}$ & $t_{4}$ \\
\hline$x_{1}$ & $x_{2}$ & $x_{3}$ & $x_{4}$ \\
$x_{2}$ & $x_{1}$ & $x_{4}$ & $x_{3}$ \\
$x_{3}$ & $x_{4}$ & $x_{2}$ & $x_{1}$ \\
$x_{4}$ & $x_{3}$ & $x_{1}$ & $x_{2}$
\end{tabular}

Table 1: An example where no type is extreme.

Note that $\phi\left(t_{i}\right)=x_{i}$ for all $i=1, \ldots, 4$. For $t_{1}$ to be extreme, the only candidate for a compromise policy against $x_{1}$ is $x_{2}$ since the former is ranked second under $t_{2}$. However, $x_{1}$ is ranked above $x_{2}$ according to $t_{4}$ and this can not be the compromise policy either. Consequently, $t_{1}$ is not an extreme type. A similar reasoning establishes that no other types are extreme. Thus all types are moderates.

In certain other cases however there is a simple characterization of extreme types. Take for instance a unidimensional Euclidean society where $X=\mathbb{R}$ and $T$ consists of finitely many real numbers $t_{1}<\ldots<t_{n}$. Further $u(x, t)=-|x-t|$. Here, the smallest type $t_{1}$ clearly represents an extreme type since the compromise policy $t_{2}$ is ranked higher than $\phi\left(t_{1}\right)=t_{1}$ by all types, (other than $t_{1}$ ). Similarly, the largest type $t_{n}$ is also extreme since $t_{n-1}$ is a compromise policy among the other types. Moreover, no other types are extreme. A similar characterization as above also holds for the "model of voice" referred to earlier. 
In fact, the implication that the end points correspond to extreme preferences holds more generally. Consider a family of preferences such that whenever a pair of types $t_{1}, t_{2}$ rank $x$ at least as high as $y$, then type $t_{3}$ which is a convex combination of $t_{1}$ and $t_{1}$, ranks $x$ as high as $y$ as well. Given such a family of preferences, it is possible to equate the types with $n$ real numbers $t_{1}<\cdots<t_{n}$. It is not necessary to assume that the policy space is unidimensional but assume that $\phi\left(t_{i}\right) \neq \phi\left(t_{j}\right)$ if $i \neq j$. We now argue that $t_{1}$ and $t_{n}$ are the only 'extreme' types. To see why this is true, consider the type $t_{1}$ and let $y=\phi\left(t_{2}\right)$ be the compromise policy. We need to show that all types other than $t_{1}$ rank $y$ strictly above $x=\phi\left(t_{1}\right)$. Suppose by way of contradiction, that there is a type, say $t$, to the right of $t_{2}$ according to which $x=\phi\left(t_{1}\right)$ is ranked at least as high as $y=\phi\left(t_{2}\right)$. Since $t_{1}$ ranks $x$ above $y$ and since $t_{2}$ is in between $t_{1}$ and $t$, the former must be ranked at least as high as the latter by $t_{2}$, a contradiction to the fact that $y$ is the favorite policy of $t_{2}$. An analogous argument establishes that $t_{n}$ is the only other extreme type.

In a number of situations it may not be possible to order types on a line in the manner described above, although it may be possible to order them in some other ways. For instance, in a Euclidean society, depending on the location of the types, they can be ordered along each coordinate keeping the other coordinates fixed. The concept of intermediate preferences due to Grandmont 1978 in fact allows a fairly general spatial ordering of preferences in higher dimensions.

Formally, $u(\cdot, \cdot)$ is said to possess the intermediate property $\left.\right|^{2}$ if for any $x, y \in \mathbb{R}^{k}$ and $s, t \in \mathbb{R}^{\ell}$ and for all $\lambda \in(0,1)$,

$$
\begin{aligned}
& u(x, s) \geq u(y, s) \text { and } u(x, t) \geq u(y, t) \\
& \Longrightarrow u(x, \lambda s+(1-\lambda) t) \geq u(y, \lambda s+(1-\lambda) t)
\end{aligned}
$$

where the inequality in the consequent is strict if at least one of the inequalities in the antecedent is also strict.

The utility function used in an Euclidean society satisfies the intermediate property. Grandmont [1978 contains a procedure for generating a family of preferences satisfying the intermediate property given a pair of utility functions. Caplin and Nalebuff [1991] provide particular examples of other families of utility functions that satisfy the intermediate property.

Grandmont 1978 itself demonstrates the powerful implications of the spatial ordering of preferences, as described by the intermediate property, for the transitivity of the majority rule and generalizations of the median voter theorems. Similar issues are pursued further in Caplin and Nalebuff [1991], Caplin and Nalebuff [1988]. In a different vein, Demange 1994 employs similar restrictions on preferences to argue the existence of stable coalition structures while Sprumont [1993] discusses the emergence of Rawlsian arbitration schemes.

Our interest in the intermediate property stems from the fact that it is a generalization of the linear structure of unidimensional types to higher dimensions. Thus, it presents a natural premise for an inquiry into whether such a society allows for a simple characterization of extreme types? For instance, is it the case that a type is extreme if and only if it cannot be expressed as a convex combination of the remaining types? After all this was the defining

\footnotetext{
${ }^{2}$ The class of intermediate preferences studied by Grandmont 1978 is in fact more general than the description used here. Among other things, Grandmont [1978 allows for non-transitive preferences while we do no.
} 
characteristic in the unidimensional case. The answer turns out to be in the negative as is illustrated in the following example.

Example 1. Consider the two dimensional Euclidean society with types $t_{1}=(0,0), t_{2}=(1,0)$ and $t_{3}=(0.5,0.5)$ and $X$ is the line joining $(0,0)$ and $(0,1)$. Note that $\phi\left(t_{3}\right)=(0.5,0)$ lies on the line joining $t_{1}$ and $t_{2}$.

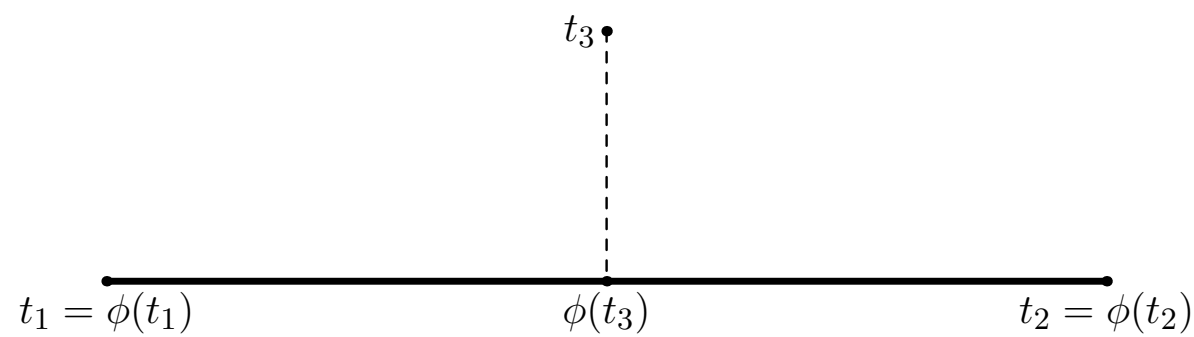

Figure 1: $t_{3}$ is not an extreme type even if it is not a convex combination of $t_{1}$ and $t_{2}$.

In this example, no one type can be expressed as a convex combination of the other two types. Yet $t_{3}$ is not an extreme type since any $x \in X$ to the left or to the right of $\phi\left(t_{3}\right)$ makes $t_{2}$ and $t_{1}$ respectively worse off. On the other hand, it is equally evident that $t_{1}$ and $t_{2}$ are both extreme types.

The key feature that separates $t_{3}$ from the remaining two types is that $\phi\left(t_{3}\right)$ happens to be the favorite policy of a type that is a convex combination of the remaining two types. As we shall show below in Theorem 1, it is precisely this property which determines whether a type is extreme or not.

First some notation. Given a compact subset $P$ of some Euclidean space, let $\operatorname{Con}(P)$ denote its convex hull. Recall that $p \in P$ is an extreme point of $\operatorname{Con}(P)$ if it cannot be expressed as a convex combination of the other elements of $P$. Let $\mathcal{E}^{*}(P)$ denote the set of all extreme points of $P$.

Given a society $(X, T, u)$, consider the following subset of $\mathcal{E}^{*}(T)$ :

$$
\mathcal{E}(X, T, u)=\left\{t \in \mathbb{R}^{\ell}: \begin{array}{l}
t \text { is an extreme point of } \operatorname{Con}(T) \text { and } \\
\phi(s) \neq \phi(t) \text { for any } s \in \operatorname{Con}(T \backslash\{t\}) .
\end{array}\right\}
$$

Theorem 1. Let $(X, T, u)$ be a society where $X$ is convex and compact, $T$ is finite, $u(\cdot, \cdot)$ is continuous and strictly concave in its first argument. Moreover assume that $u(\cdot, \cdot)$ satisfies the intermediate property. Then a type is extreme if and only if it is an element of $\mathcal{E}(X, T, u)$.

Proof. See Appendix.

Note that $\mathcal{E}(X, T, u)$ can be a strict subset of $\mathcal{E}^{*}(T)$. In Example 1 , $t_{3}$ is not in $\mathcal{E}(X, T, u)$ although it is in $\mathcal{E}^{*}(T)$. There are many interesting cases however when these two sets coincide. For instance, $\mathcal{E}(X, T, u)=\mathcal{E}^{*}(T)$ in Euclidean societies where $T \subseteq X$ or in the "model of voice" of Banerjee and Somanathan 2001. For all such societies, the following is immediate. 
Corollary 1.1. Let $(X, T, u)$ be a society such that $\mathcal{E}(X, T, u)=\mathcal{E}^{*}(T)$. Also assume that the other hypotheses of Theorem 1 hold. Then a type is extreme if and only if it cannot be expressed as a convex combination of the remaining types.

Proof. Immediate.

\section{$2.1 \quad$ Infinitely Many types}

A critical assumption in Theorem 1 is that there are only finitely many types in the society. In this section we shall briefly describe how our results extend to societies with infinitely many types. A preliminary observation is that the definition of an extreme type is too strong and rules out the existence of such types even in many intuitive situations. To see this reconsider a unidimensional Euclidean society where $X$ is the set of reals, $u(x, t)=-|x-t|$ but now the set of types is the entire interval $[0,1]$. In this environment it is reasonable to think of 0 and 1 as extreme types. However, there is no policy which makes every other type strictly better off relative to the policy $\phi(0)=0$. Therefore 0 cannot be an extreme type. A similar argument rules out 1 as being an extreme type.

For a definition that conforms with the intuition and is applicable at least in the more common modelling practices, we settle for the following weakening of the notion of what it means to be an extreme type.

Definition (Virtually Extreme Types). Given a society $(X, T, u)$, a type $t \in T$ is said to be virtually extreme if it is extreme in every society $(X, S, u)$, where $S$ is a finite subset of $T$ and contains $t$.

That is, a type is virtually extreme among a family of preferences if it is an extreme type in every finite sub-family that contains it.

Theorem 2. Let $(X, T, u)$ be a society where $X$ is convex and compact, $T$ is compact $u(\cdot, \cdot)$ is continuous and strictly concave in its first argument. Moreover assume that $u(\cdot, \cdot)$ satisfies the intermediate property. Then a type is virtually extreme if and only if it is an element of $\mathcal{E}(X, T, u)$.

Proof. See Appendix.

\section{$2.2 \quad$ Lotteries and Extreme preferences}

In many situations, it is common to model the social decision as a lottery over a set of sure policies. Allowing for randomization enlarges the set of potential compromises. Such possibilities may allow a compromise against an otherwise moderate type and render her extreme. Of course, introduction of lotteries will not have an effect on a type that is already considered extreme in their absence. What we now argue is that under the assumption of the expected utility hypothesis, one can continue to apply Theorem 1 and Theorem 2 to the set of sure policies by taking the utility function $u(\cdot, \cdot)$ in those theorems to denote the vNM utility functional and strengthen the assumption of quasi-concavity to concavity. 
To elaborate, suppose that the set of policies available to a society is $\Delta(X)$ which is the set of lotteries over a set of pure policies $X$ and $u(x, t)$ is the vNM utility of type $t$. The utility of policy, say $p(\cdot)$, which is now a probability measure over $X$, is

$$
V(p, t)=\int_{x \in X} u(x, t) \mathrm{d} p(x)
$$

Assume that the hypotheses of Theorem 1 or Theorem 2 apply for the tuple $(X, T, u)$ except that $u(\cdot, t)$ is now strictly concave so that individuals are risk averse. By the Jensen's inequality, the favorite lottery of $t \in T$ is in fact a sure outcome $\phi(t) \in X$. Therefore if $t$ is an extreme type, there is a policy $p(\cdot)$ which all the types other than $t$ strictly prefer to the policy $\phi(t)$. However, note that due to concavity of $u(\cdot, s)$, the inequality $V(p, s) \leq u\left(E_{p}(x), s\right)$ must hold for all $s \neq t$. Consequently, the sure outcome $E_{p}(x)$ would also be a compromise policy against $t$. In other words, if it is possible to find a compromise against $t$ when lotteries over $X$ are permitted, then it is also possible to do so even when lotteries are not available.

The applicability of Theorem 1 discussed above plays an important role in our analysis of Section 3 ,

\section{Active Lobbying and Extreme Preferences}

In this section, we consider a simple model of rent seeking and discuss the pattern of lobbying activity with regard to the characteristics of the agents preferences.

Consider a society $(X, T, u)$ where $T=\left\{t_{1}, \ldots, t_{n}\right\}$ with $\mathcal{P}_{i}$ agents of type $t_{i}$. The vNM utility of an agent of type $t \in T$ for a policy $x \in X$ is $u(x, t)$. We will assume that $u(\cdot, \cdot)$ is concave in both its arguments.

Players seek to influence the social choice in their favor by spending resources. Contributions are made simultaneously. Although a player may to contribute to more than one cause, we avoid unnecessary mathematical complications by restricting contributions to only finitely many policies. A player is however free to choose which finite subset of polices that she wishes to contribute.

A strategy is then a function $r: X \longrightarrow \mathbb{R}_{+}$with the property that $r(x)>0$ for at most finitely many $x$. Let $\bar{r}$ denote her total contribution from the strategy $r(\cdot)$. Given the choices of the individual players, let the function $R: X \longrightarrow \mathbb{R}_{+}$describe the aggregate contributions so that $R(x)$ is the total amount contributed by all the players for the policy $x$.

Given a strategy profile, consider the set of policies which receive a positive contribution. Denote this finite set by $Q=\left\{x_{1}, \ldots, x_{m}\right\}$ so that $R(x)>0$ if and only if $x \in Q$. Note that $Q$ depends on the strategy profile although we will not make this dependence explicit. The aggregate resources spent on all the policies taken together is a simple sum namely $\bar{R}=\sum_{x \in Q} R(x)$.

We capture the decision process as a lottery over $X$ as follows. Given a strategy profile, the policies are chosen as per the following lottery:

$$
p(x)=\left\{\begin{array}{cc}
\frac{R\left(x_{i}\right)}{\bar{R}} & \text { if } x_{i} \in Q \\
0 & \text { otherwise } \\
8 &
\end{array}\right.
$$


unless of course $R(x) \equiv 0$. If $R(x) \equiv 0$, then policies are drawn according to a given arbitrary but fixed density on $X$.

Modelling the collective decision process as the above reduced form follows a vast literature on public choice at least since Tullock 1980]. Other notable applications include Becker 1983] and Grossman [1991]. Esteban and Ray [1999] contains a number of other references to works that employ the above reduced form in varied applications.

Let $g(\rho, t)$ be the opportunity cost of a type $t$ player for committing an amount $\rho$ toward influencing activity. The payoff of a player of type $t$ who contributes according to $r(\cdot)$ while aggregate contributions are given by $R(\cdot)$ is

$$
V(r, t)=\sum_{x \in Q} u\left(x_{i}, t\right) p\left(x_{i}\right)-g(\bar{r}, t)
$$

unless $R(x) \equiv 0$. If $R(x) \equiv 0$ then the payoff is simply some constant.

The above completes a description of a game in strategic form, which in the sequel we refer to as the lobbying game. Note that the lobbying game is identical to the model studied in Esteban and Ray 1999 except in one critical respect. Here, each individual player is free to choose how much she wants to contribute. In contrast, Esteban and Ray 1999 views individuals as members of different groups and the group as a whole chooses the contribution for each member. Such a specification glosses over issues of free-riding problems articulated ${ }^{3}$ in Olson 1965].

For the usual reasons, namely to ensure that the payoff function is concave and to employ calculus methods to determine the best response, we shall assume that for each $t \in T$, the cost function $g(\cdot, t)$ is convex and twice differentiable. We shall use $g^{\prime}(\cdot, t)$ to denote the partial derivative of $g(\cdot, \cdot)$ with respect to its first argument - which is simply the marginal cost of contributing resources for a type $t$ player.

Observe that given the lobbying rules described by (3), if none of the players were to contribute, then a typical player would find it profitable to contribute a small amount to her favorite policy. For, such an action would ensure that her favorite policy is picked with probability one and yields an infinite marginal benefit at the marginal cost of $g^{\prime}(0, t)$. Consequently, in any equilibrium, one must necessarily have $\bar{R}>0$. This should be kept in mind in the subsequent discussion since we routinely divide by $\bar{R}$.

Proposition 1. A Nash equilibrium of the lobbying game exists. Moreover, in any Nash equilibrium, a player either contributes a positive amount to her ideal policy or does not make a contribution.

Proof. See Appendix.

In presenting our results below, we appeal to Theorem 1 and Corollary 1.1. These results do not (explicitly) involve lotteries, unlike in the present lobbying game. One must therefore bear in mind the discussion in Section 2.2 in interpreting Theorem 3 and Corollary 3.1 below.

Theorem 3. Consider a society $(X, T, u)$ that satisfies the hypotheses of Corollary $1.1, u(\cdot, \cdot)$ is strictly concave and for each $t \in T$ and $u(\phi(t), t)$ is a constant $\bar{u}$. Also assume that $g^{\prime}(0, t)$

\footnotetext{
${ }^{3}$ See Esteban and Ray 2001. however,
} 
is a positive constant $c$. Then there exists an integer $M$ such that whenever the economy consists of at least $M$ agents of each type, any agent who makes a contribution must be an extreme type.

Before we turn to the main arguments in the above proof, several remarks are in order.

First, when the marginal cost of contribution is a constant, the minimum number of players of each type $M$ in the theorem can be taken to be one. Second, it is important to note that in equilibrium, there may exist some extreme types who remain inactive. The Theorem only rules out moderates making positive contribution in equilibrium 4 . When the type space is unidimensional, since it cannot be an equilibrium for only one type to make a contribution, we have the following stronger result.

Corollary 3.1. Suppose $T$ is unidimensional and all other hypotheses of Theorem 3 are satisfied. If there are sufficiently large number of players of each type, then in every equilibrium exactly two types of players engage in lobbying activity. These are the players corresponding to the two extreme types.

It is interesting to compare the above observation for unidimensional types with a result in Esteban and Ray [1999] which shows that 'conflict' in the society (as measured by the sum total of equilibrium contributions) is maximized when there are exactly two symmetric groups. In their work, they do not allow for voluntary contributions but the lobby groups choose the level of contribution for each player and study comparative statics exercises. In this model, with the possibility of players making voluntary contributions, the above shows that the bimodal outcome is the only equilibrium outcome in a large economy.

Third, the conclusion of Theorem 3 that only extreme types are active does not necessarily extend to societies that satisfy only the hypotheses of Theorem 1 and but not that of Corollary 1.1, as the following example shows.

Example 2. Consider a two dimensional Euclidean society where $t_{1}=(-1,0), t_{2}=(2,0)$ and $t_{3}=(0.5,1)$ and $X$ is the line segment joining $(0,0)$ and $(1,0)$. Here $\phi\left(t_{1}\right)=(0,0)$, $\phi\left(t_{2}\right)=(1,0)$ and $\phi\left(t_{3}\right)=(0.5,0)$. No type can be expressed as a convex combination of the other two. Therefore, $\phi\left(t_{3}\right)$ is however the favorite policy of type $(0.5,0)$ which is a convex combination of types $t_{1}$ and $t_{2}$, which means $\mathcal{E}(X, T, u) \neq \mathcal{E}^{*}(T)$, Corollary 1.1 does not apply. Theorem 1 however, can be applied to conclude that $t_{3}$ is a moderate. Now note that $\bar{u}=1$ as each type is at a distance of 1 from $X$. Take $g(\rho, t)=0.25 \rho$ so that all other hypotheses of Theorem 3 are met. Then, using symmetry, it straightforward to support an equilibrium where all three types contribute equal amount:5.

Finally, we note since the outcome of the lobbying game above is modelled as a lottery over outcomes that receive positive contributions, the implication of Proposition 1 and Theorem 3 is that in equilibrium, it is only the ideal policies of the extreme types that are implemented. One might argue that even though agents lobby for their ideal policies, the actual outcome

\footnotetext{
${ }^{4}$ It suffices to consider a two dimensional Euclidean society for an example of when this is true. Take $X$ to be the triangle with vertices $x_{1}=(0,0), x_{2}=(1,0)$ and $x_{3}=(0.5, \epsilon)$ for a small positive $\epsilon>0$. If we now take $T=\left\{x_{1}, x_{2}, x_{3}\right\}$, for $\epsilon$ sufficiently small, the type $x_{3}$ cannot be active, even though it is an extreme type.

${ }^{5}$ Similar counter examples can be can be constructed to show that none of the other hypotheses in Theorem 3 is superfluous.
} 
instead of being a lottery is a compromise of these different policy positions. In fact, Osborne et al. [2000] study a model of collective decision making in committees where the compromise rule is not a lottery but some "average" outcome.

One main conclusions are unaffected for certain modifications of the lobbying game so that the eventual policy is taken to be such an "average policy". Indeed, modify the lobbying game so that given a vector of aggregate contributions $R$, the average policy

$$
\bar{x}=\sum_{x_{i} \in Q} p\left(x_{i}\right) x_{i}
$$

is chosen. As before, if $R(x) \equiv 0$, then policies are drawn according to a given arbitrary density on $X$. Assume, as they do, that the utility of $\bar{x}$ to a type $t$ is

$$
V(r, t)=u(\|\bar{x}-t \mid\|)-g(\bar{r}, t)
$$

where $g(\bar{r}, t)$ as before denotes the opportunity cost of committing an amount $\bar{r}$ toward influencing activity and $u(\cdot)$ is some concave function.

We shall refer to game implied by the above as the modified lobbying game.

The modified lobbying game is very similar to the one used by Osborne et al. 2000]. A major difference is that in their model an individual can only choose whether to participate in the committee. Here an individual has the possibility of expressing the intensity of her preference by varying the amount of resources they spend on influencing the policy.

Following the methods used in the proofs of Proposition 1 and Theorem 3 the following can be proved. We omit its proof.

Proposition 2. Consider a Euclidean society $(X, T, u)$ where $T \subseteq X$. The modified lobbying game admits an equilibrium. Moreover, there exists an integer $M$ such that whenever the economy consists of at least $M$ agents of each type, any agent who contributes a positive amount is necessarily an extreme type.

Proof of Theorem 3. We present here the arguments for the case when the marginal cost of contributions is a constant, that is $g(\rho, t)=c \rho$ for all $\rho \geq 0$ and for all $t$. The case of increasing marginal costs and any other missing details can be found in the Appendix.

When the marginal cost is a constant, it suffices to take $M=1$ so that the moderates never contribute regardless of population sizes. From Proposition 1, in an equilibrium an agent either contributes a positive amount to her favorite policy or not at all. Therefore the aggregate contributions to different policies can be represented by a vector $R=\left(R_{1}, \ldots, R_{n}\right)$, where $R_{i}$ is the amount spent on policy $\phi\left(t_{i}\right)$. In an equilibrium, $\bar{R}=\sum_{i} R_{i}>0$ so that $\phi\left(t_{i}\right)$ is chosen with probability $p_{i}=R_{i} / \bar{R}$ and no other policy is chosen.

Define

$$
\operatorname{mb}(R, t)=\frac{1}{\bar{R}} \sum_{i=1}^{n} p_{i}\left[\bar{u}-u\left(\phi\left(t_{i}\right), t\right)\right]
$$

It turns out ${ }^{6}$ that $\operatorname{mb}(R, t)$ is the marginal benefit of a player of type $t$ from contributing to her ideal policy in an equilibrium where $R$ is the vector of aggregate contributions. Clearly,

\footnotetext{
${ }^{6}$ See the derivation of Eq. 8 in the Appendix and conclude 4
} 
for $R$ to constitute an equilibrium, this marginal benefit must be no greater than the marginal cost of making a contribution, which is to say that

$$
\operatorname{mb}(R, t) \leq c \quad \forall t \in T
$$

with the above being an equality whenever $R_{t}>0$.

Since $u(\cdot, \cdot)$ is strictly concave, $\operatorname{mb}(R, \cdot)$ is strictly convex in its second argument. Therefore the maxima of $\operatorname{mb}(R, \cdot)$ occur only at exposed points of $T$. For all other types, (5) holds as a strict inequality. Consequently if at all a type makes a contribution, then she is an exposed point $T$. Applying Theorem 1 yields the result.

To prove the general case of increasing marginal costs, we first show that an implication of convex costs is that even if the population is very large, the total amount spent on lobbying activity in any equilibrium is bounded. Therefore, for large enough population sizes, the contribution of an individual player is close to zero. Then the relation (5) with $c=g^{\prime}(0, t)$ must be approximately true. This is enough to repeat the arguments for the case of the constant marginal cost the conclude that the above cannot hold as an equality for a moderate type. Missing details can be found in the formal proof in the Appendix.

\section{A Proofs of Results in Section 2}

Proof of Theorem 1. Our arguments involve extensive use of Proposition Section 3, Grandmont 1978 which we state below as Result 1 (in a form convenient for our analysis).

Result 1 (Grandmont [1978]). Suppose $u(\cdot, \cdot)$ satisfies the intermediate property. Then for each $x \neq y, x, y \in X$, there exists a unique hyperplane $H(x, y) \subseteq \mathbb{R}^{\ell}$ such that the types that strictly prefer $x$ to $y$ lie above $H(x, y)$, the types that are indifferent to $x$ and $y$ lie on $H(x, y)$ while the remaining types lie below $H(x, y)$.

Suppose $t$ is an extreme type and let $x$ be a compromise policy against $t$. Then by Result 1 every type in $T$ other than $t$ lies below the hyperplane $H(\phi(t), x)$ while $t$ lies above it. This implies two things. First $t$ is an extreme point of $T$. Second, Con $(T \backslash\{t\})$ lies below $H(\phi(t), x)$. Therefore any type $s \in \operatorname{Con}(T \backslash\{t\})$ also prefers $x$ to $\phi(t)$, which in turn means $\phi(s) \neq \phi(t)$.

Now for each $s \in T \backslash\{t\}$, let $\gamma(s)=(1-\epsilon) s+\epsilon t$ and let $K=\operatorname{Con}\{\gamma(s): s \in T \backslash\{t\}\}$. We will exhibit a policy $x$ such that for a suitably chosen positive $\epsilon$, every type in $K$ weakly prefers $x$ to $\phi(t)$, so that $K$ lies on or below the hyperplane $H(\phi(t), x)$. Since $t$ of course lies above $H(\phi(t), x)$, by construction then $T \backslash\{t\}$ lies strictly below $H(\phi(t), x)$ from which we conclude that $x$ is a compromise policy against $t$.

Choose any $s \in \operatorname{Con}(T \backslash\{t\})$. Since $\phi(s) \neq \phi(t)$, by the intermediate property, there exists a (unique) type $s^{\prime}$, which strictly in between $s$ and $t$ on the line joining them such that all the types between $s$ and $s^{\prime}$ strictly prefer $\phi(s)$ to $\phi(t)$. This in particular implies that $\phi(t)$ is not the favorite policy of any of these types. Consequently, we can choose an $\epsilon$ sufficiently small but positive so that

$$
\phi(s) \neq \phi(t) \quad \forall s \in K .
$$

It remains to exhibit a policy $x$ which the types in $K$ unanimously weakly prefer to $\phi(t)$. Using (6), we do this below. 
Let $K^{*}=\left\{s_{1}, \ldots, s_{m}\right\}$ denote the extreme points of $K$. For $i=1, \ldots, m$, let $F_{i}=\{s \in$ $K: u\left(\phi\left(s, s_{i}\right) \geq u\left(\phi(s), s_{i}\right)\right\}$. In words, $F_{i}$ is the set of types whose favorite policies type $s_{i}$ weakly prefers $\phi(t)$. Now suppose $S \subseteq K^{*}$ and $s \in \operatorname{Con}(S)$. If $u\left(\phi(s), s_{i}\right) \leq u\left(\phi(t), s_{i}\right)$ for all $s_{i} \in S$, then by the intermediate property $u(\phi(s), s) \leq u(\phi(t), s)$ in violation of $(6)$. Therefore there exists $s_{i} \in S$ such that $s \in F_{i}$. Since $S$ was chosen to be an arbitrary subset of $S$, we have just shown that

$$
\operatorname{Con}(S) \subseteq \cup_{s_{i} \in S} F_{i} \quad \forall S \subseteq K^{*}
$$

Noting that each $F_{i}$ is closed, a direct application of the K.K.M Lemma 7 shows that $\cap_{s_{i} \in K^{*}} F_{i} \neq$ $\emptyset$. For an $x \in \cap_{s_{i} \in K^{*}} F_{i}$ the set $K^{*}$ lies on or below $H(\phi(t), x)$ which in turn implies that $K$ must lie on or below this hyperplane.

Proof of Theorem 2. Suppose $t \in \mathcal{E}(X, T, u)$. Then clearly $t \in \mathcal{E}(X, S, u)$ for any finite $S \subseteq T$ that contains $t$. By Theorem $1, t$ is an extreme type in $(X, S, u)$.

Conversely, suppose $t$ is a virtually extreme type. If $t$ is not an extreme point of $\operatorname{Con}(T)$, by the Caratheodary Theorem, there exists finite subset $S$ of $T$ that does not contain $t$ but $t \in \operatorname{Con}(S)$. Therefore $t$ is not an extreme point of $\operatorname{Con}(S \cup\{t\})$ and by Theorem 11, it is not an extreme type of the society $(X, S \cup\{t\}, u)$. To complete the proof that $t \in \mathcal{E}(X, T, u)$, repeat the arguments found in the "if" part of proof of Theorem 1 to conclude that $\phi(t)$ cannot be the favorite policy of any type in $\operatorname{Con}(T \backslash\{t\})$.

\section{B Proofs of Results in Section 3}

We begin by seeking equilibria in which all players of a given type contribute the same amount and only to her favorite policy. A strategy profile can then be described by a vector $r=\left(r_{1}, \ldots, r_{n}\right)$ where $r_{i}$ is the contribution of a type $t_{i}$ player toward her ideal policy.

Lemma 1. In any equilibrium, a player either contributes to her favorite policy or not at all. Moreover, the following is a necessary and sufficient condition for $r=\left(r_{1}, \ldots, r_{n}\right)$ to constitute an equilibrium.

$$
\operatorname{mb}\left(R, t_{i}\right) \leq g^{\prime}\left(r_{i}, t\right) \quad \forall t_{i} \in T
$$

where $\operatorname{mb}(R, t)$ is as defined in (4) and the above holds as equality provided $r_{i}>0$.

Proof. Let $Q=\left\{x_{1}, \ldots, x_{m}\right\}$ contain the subset of policies that receive a positive contribution for a given strategy profile and let $R=\left(R_{1}, \ldots, R_{m}\right)$ be the corresponding vector of aggregate contributions to these policies. Given $R$, and an arbitrary policy $y$, the expected benefit of a player of type $t$ can be written as

$$
B=u(y, t)-\sum_{x_{i} \in Q} p_{i}\left[u(y, t)-u\left(x_{i}, t\right)\right]
$$

Straightforward differentiation readily yields the marginal benefit of contributing to an arbitrary policy $y$ for a player to type $t$ is given by

$$
\frac{1}{\bar{R}} \sum_{x_{i} \in Q} p_{i}\left[u(y, t)-u\left(x_{i}, t\right)\right]
$$

\footnotetext{
${ }^{7}$ See Chapter 5, Border 1985 for example.
} 
which clearly achieves a unique maximum at $y=\phi(t)$. Consequently, a type $t$ player must only contribute to her favorite policy. The condition (7) is merely the necessary first order condition for $r_{i}$ to be a best response, which is also sufficient by virtue of the fact that the payoff function is concave.

Proof of Proposition 1. Pick any $\left(\beta_{1}, \ldots, \beta_{n}\right)$ such that $\beta_{i}>0$ and $\sum_{i=1}^{n} \beta_{i}=1$ and $k>0$. Now modify the lobbying game presented in the body of the paper as follows. First the strategy of a player is restricted to making a contribution, if at all, to her favorite policy. Second, if $R=\left(R_{1}, \ldots, R_{n}\right)$ are the vector of aggregate contributions, then policy $t_{i}$ is chosen with probability

$$
p_{i}^{k}=\frac{k \beta_{i}+R_{i}}{k+\bar{R}}
$$

where $\bar{R}=\sum_{i} R_{i}$. At $R$, the expected utility of a player of type $t_{i}$ who has contributed an amount $\rho \geq 0$ is given

$$
\begin{aligned}
U(\rho, t) & =\sum_{i=1}^{n} p_{i}^{k} u\left(t_{i}, t\right)-g(\rho, t) \\
& =\bar{u}-\sum_{i=1}^{n} p_{i}^{k}\left[\bar{u}-u\left(t_{i}, t\right)\right]-g(\rho, t)
\end{aligned}
$$

We will refer to the above game as the perturbed lobbying game where $k$ is the size of the perturbation. We will first argue that this game admits an equilibrium and the show that an appropriate limit of these equilibria as $k \rightarrow 0$ constitutes an equilibrium of the original game.

The payoff functions of the $k$-perturbed are strictly concave and unlike in the unperturbed game, continuous. Through standard arguments, an equilibrium exists for the $k$-perturbed game exists. It will be evident from the first order conditions presented below that every player of a given type contributes the same amount, provided $g^{\prime \prime}(\rho, t)>0$. When $g^{\prime \prime}(\rho, t)=0$, the marginal cost of contribution is a constant and in this case there will be no loss in generality in assuming that players of the same type choose an identical contribution. Hence $r=\left(r_{1}^{k}, \ldots, r_{n}^{k}\right)$ were $r_{i}^{k}$ is the contribution of an agent of type $t_{i}$ adequately describes an equilibrium. Let $R^{k}=\left(R_{1}^{k}, \ldots, R_{n}^{k}\right)$ denote the vector of aggregate contributions and $\bar{R}^{k}=$ $\sum_{i} R_{i}^{k}$. Differentiating $U(\cdot, t)$ with respect to the first argument, the following first order condition must hold in equilibrium:

$$
\frac{1}{k+\bar{R}^{k}} \sum_{j=1}^{n} p_{j}^{k}\left[\bar{u}-u\left(t_{j}, t_{i}\right)\right] \leq g^{\prime}\left(r_{i}^{k}, t_{i}\right) \quad \forall t_{i} \in T
$$

where the above is an equality if $r_{i}^{k}>0$. Three useful properties of the equilibrium follow from the first order condition (9). First, we note that $r^{k}=\mathbf{0}$ cannot constitute an equilibrium when $k$ is sufficiently small. For if this where the case, the above LHS shows that the marginal benefit of a player of type $t$ is $\sum_{i=1}^{n} \beta_{i}\left[\bar{u}-u\left(t_{i}, t\right)\right] / k$, which is positive. For $k$ sufficiently small, this is higher than the marginal cost of making a small contribution, namely $g^{\prime}(0)$. From this we can conclude that $r_{i}^{k}=0$ cannot be a best response and thus obtain a contradiction. In the remainder of this proof, we restrict attention to such values $k$.

Second, $\left\{r_{i}^{k}\right\}_{k}$ must be a bounded sequence for each $t_{i} \in T$. For if this were not the case for some $t_{i}$, then the LHS of (9) would converge to zero while the RHS, due to the convexity $g_{i}(\cdot)$, would remain positive. Without loss of generality, we shall assume that $\lim _{k \rightarrow 0} r_{i}^{k}=r_{i}^{*}$ is well 
defined for all $t_{i}$. (Since $\left\{r_{i}^{k}\right\}$ is bounded, we could otherwise pick a suitable subsequence for which this is true). Consequently, $\lim _{k \rightarrow 0} R^{k}=R^{*}$ is also well defined.

Third, $R^{*}>0$. This must be true since (9) must hold as an equality for at least one player for each $k$ since $\bar{R}^{k}>0$. As there are only finitely many types, without loss of generality, there exists a $t_{i}$ such that $(9)$ holds as an equality along the entire sequence 8 . Putting together the above three observations, and taking the appropriate limits with respect to $k$ in (9) shows that $r^{*}=\left(r_{1}^{*}, \ldots, r_{n}^{*}\right)$ satisfies $(7)$. The proof is complete on applying Lemma 1

Proof Of Theorem 3. Assume $g^{\prime \prime}(\rho, t)>0$ as the case when $g^{\prime}(0, t)=0$ has already been covered in the main body of the text. Assume, by way of contradiction that no much $M$ exist. Then there exists an infinite sequence $\left\{M_{k}\right\}_{k=1}^{\infty}$ such that (a) $\lim _{k \longrightarrow \infty} M_{k}=\infty$ and (b) for each $k$, there exists a configuration of population sizes $\left\{\mathcal{P}_{i, k}\right\}$ such that $\mathcal{P}_{i, k} \geq M_{k}$ and an equilibrium $r^{k}=\left(r_{1}^{k}, \ldots, r_{n}^{k}\right)$ in which at least one moderate type that is active. As there are only finitely many types, it is possible to pick, one moderate type $t_{i}$ such that $r_{i}^{k}>0$ for all $k$ and $\operatorname{mb}\left(R^{k}, t_{i}\right)=g^{\prime}\left(r_{i}^{k}, t\right)$. Since $g^{\prime}(0, t)>0$, it follows from the above equality that the sequence of aggregate contributions $\left\{\bar{R}^{k}\right\}$ is bounded, and hence, without loss of generality, converges to say $R^{*}$. Since total contribution are bounded, it follows that $\lim _{k \rightarrow \infty} r_{j}^{k}=0$ for all $t_{j} \in T$. Consequently, we have

$$
\operatorname{mb}\left(R^{*}, t_{j}\right) \leq g^{\prime}\left(0, t_{j}\right) \quad \forall t_{j} \in T
$$

with the above holding as an equality for the moderate type $t_{i}$. Repeat the arguments provided in the body of the paper in the case of constant marginal cost to arrive at a contradiction.

\section{REFERENCES}

Abhijit Banerjee and Rohini Somanathan. A simple model of voice. Quarterly Journal of Economics, 116(1):189-227, 2001.

Gary Becker. A theory of competition among pressure groups for political influence. Journal of Political Economy, 98:371-400, 1983.

Duncan Black. The Theory of Committees and Elections. Cambridge University Press, Cambridage, 1958.

Kim C. Border. Fixed Point Theorems with Applications to Economics and Game Theory. Cambridge University Press, Cambridage, 1985.

Andrew Caplin and Barry Nalebuff. Aggregation and social choice: A mean voter theorem. Econometrica, 59(1):1-23, 1991.

Andrew S Caplin and Barry J Nalebuff. On the 64\%-majority rule. Econometrica, 56(4): 787-814, 1988.

Gabrielle Demange. Intermediate preferences and stable coalition structures. Journal of Mathematical Economics, 23(1):45-58, 1994.

\footnotetext{
${ }^{8}$ If such a type does not exist along the original sequence, one could choose a suitable subsequence for which it is true.
} 
Joan Esteban and Debraj Ray. Conflict and distribution. Journal of Economic Theory, 87 (2):379-415, August 1999.

Joan Esteban and Debraj Ray. Collective action and the group size paradox. American Political Science Review, 95(3):663-72, September 2001.

Jean-Michel Grandmont. Intermediate preferences and the majority rule. Econometrica, 46 (2):317-30, 1978.

Herschel Grossman. A general equilibrium model of insurrections. American Economic Review, 81:912-921, 1991.

Susanne Lohmann. Signalling model of informative and manipulative political action. American Political Science Review, 87(2):319-333, June 1993.

Mancur Olson. The Logic of Collective Action: Public Goods and the Theory of Groups. Harvard University Press, Cambridge, MA, 1965.

Martin J Osborne, Jeffrey S Rosenthal, and Matthew A Turner. Meetings with costly participation. American Economic Review, 90(4):927-43, September 2000.

Yves Sprumont. Intermediate preferences and rawlsian arbitration rules. Social Choice and Welfare, 10(1):1-15, 1993.

Gordon Tullock. Toward a theory of the rent seeking society. chapter Efficient Rent Seeking, pages 97-112. Texas A \& M University Press, 1980. 